\title{
Forest fires, sandpiles and the like
}

\begin{abstract}
An elegant argument about the behaviour of a simple model of a forest fire has now been extended to make connections with other systems that keep their original organization in spite of external disturbances.
\end{abstract}

ON the principle that science is the art of the possible, in Medawar's phrase, the attribute of a physical model that matters most is that it should be tractable, not that it should rigorously embody what is known of the real world. Everybody knows that, for which reason the marvel is that so many models that incorporate only crude approximations to what is expected to happen on a microscopic scale nevertheless yield good or at least suggestive approximations to reality.

Forest fires are a case in point. Just over two years ago, Per Bak and Kan Chen from the Brookhaven National Laboratory and Chao Tang from the University of California, Santa Barbara, put forward a simple model of the process in which forest fires form (Phys. Lett. A. 147, 297; 1990). It may be recalled that Bak and Tang (with $\mathrm{K}$. Wiesenfeld) were the authors, in 1987, of the intriguing analysis of the stability of a sandpile in which they showed, among other things, that such a system displays what they called "self-organized criticality"; within reason, however sand is added to a growing pile, and whatever avalanches are as a consequence induced on its surface, the result will be a profile of constant shape determined only by the microscopic properties of the sand grains.

But how to model a forest fire? The starting point is a square lattice on a twodimensional surface. At each lattice point, there may be a tree or a vacancy for a tree. Some trees may be on fire, others are still green. The evolution of the fire is then followed by means of successive finite steps in time (which makes the model a cellular automaton in the technical sense), and by using a few simple rules to get from one time step to the next. The basic rules are these: a burning tree will have disappeared by the next time step, but will have set light to all the trees that were originally its nearest neighbours.

If this were all, the model would be very dull. In a densely packed forest, a single burning tree would eventually set light to all the others, and the region would be left free of trees. But firebreaks extending the whole breadth of the region would confine the conflagration to one part of the forest only. But in a sparsely planted forest, isolated trees would remain alive after the fire had burned itself out elsewhere. But this model of reality is dull because it fails to allow for what happens in real forests - the emergence of new trees where there is room for them. Whence the third rule for the evolu- tion of a forest, the assumption that at each time stage, there is a probability $p$ that a new tree will emerge at any vacant site.

Plainly, with these rules, if $p$ is sufficiently small, the forest fire will burn itself out (but firebreaks will not, on this occasion, leave some parts of the forest intact). What the authors were concerned to show is that when $p$ exceeds some critical value, the concentration of trees on fire will eventually reach a steady state in which, for example, it is possible to calculate (from numerical simulations) the number of trees on fire within a distance $r$ of a particular tree which is itself on fire. Nobody will be surprised that the outcome is a fractal. The number distribution increases with the radius $r$, but less quickly than $r^{2}$, the area involved.

For what it is worth, the model should serve well to represent not simply the burning of a forest but the spread of infectious diseases in a community. The original objective of the argument and the simulation that accompanies it was to throw some light on the phenomenon of turbulence, where the correlation of motions over distance is also fractal in nature. But the original paper is suggestive only.

The argument has now been carried further by B. Drossel and F. Schwabl from the Technical University at Munich (Phys. Rev. Lett. 69, 1629; 1992), who argue that the behaviour of a burning forest is more complicated and more interesting when the probability, $p$, of the emergence of new trees is small and even tends to zero. Although Bak and his associates had concluded that, in these conditions, the correlation length of burning trees (or the average distance between one burning tree and all others in the same plight) would increase indefinitely to infinity, it has since been shown by numerical simulation that there emerges a spiral pattern of fire-fronts whose dimensions are of the order of $1 / p$, the inverse of the growth rate of the forest.

Drossel and Schwabl even have a simple explanation of the phenomenon. When $p$ is small and if there is a steady state, most of the underlying forest lattice will have been emptied of trees. The result will be that clusters of new growth will emerge in the cleared ground and, by their isolation, will remain free from fire until the cluster to whch they belong has grown to merge with another in which there are burning trees. Accordingly, burning trees will invariably be included within large clusters which also include trees not yet set alight, and whose diameter will be of the order of $1 / p$, whence the dimensions of the fire-fronts found in the numerical simulations. The system is not critical in the sense that the correlation length does not diverge more quickly than the controlling parameter.

So how to make the burning forest critical in this technical sense? Some way must obviously be found to ignite the trees that stand in isolated clusters. What better than lightning strikes, which may destroy intact trees by a random process like that which allows the emergence of new trees at cleared sites? Drossel and Schwabl show that, if the probability of a lightning strike at a particular site is $f$ per time step, the properties of the system as a whole are entirely determined by the ratio $f / p$.

The elegance of the continuation of this argument is that it derives most of the other properties of burning forests, made even more realistic by allowing that even burning trees do not disappear instantaneously, but that their demise is spread over several time steps, by simple dimensional arguments, which are confirmed by simulation. One of the conclusions, for example, is that the burning of a forest will correspond to self-organized critical behaviour if the time in which a cluster of trees burns down (of the order of the square root of $(f / p)$ in the two-dimensional case) is less than the growth rate of the trees (proportional to $(1 / p))$, which must in turn be less than $(1 / f)$, essentially the interval of time between successive lightning strikes at the same site.

It seems unlikely that these conclusions will be of immediate benefit to foresters and such people, although the parellels with the behaviour of real forest fires should be readily apparent in the way in which successive waves of fire seem to be entirely compatible with the maintenance of a forest in something like a steady state. The more interesting analogy is with the sandpile problem. What Drossel and Schwabl show for the forest case is that critical behaviour requires that the time for clusters of trees to burn down must be less than that required for them to grow individually. The sandpile analogue is that the slumping of an avalanche induced by the accumulation of material randomly is faster than the rate at which material is added to the growing pile. One of these days, it may even be possible to connect this argument with the urgent and practical business of turbulence.

John Maddox 